\title{
An Artificial Intelligence Approach to Predict Different Strokes
}

\author{
Ankitha $\mathrm{S}^{1}$, Deepthi $\mathrm{M}^{1}$, Harshavardhan $\mathrm{N}^{1}$, Remanth $\mathrm{M}^{1}$, Janhavi $\mathrm{V}^{2}$ \\ ${ }^{1,2}$ Department of Computer Science and Engineering \\ Vidyavardhaka College of Engineering, Mysuru, \\ Karnataka, India
}

\begin{abstract}
Stroke is the subsequent driving reason for death, and was a serious, long haul inability. Stroke is the unexpected passing of cerebrum cells because of oxygen misfortune brought about by hindering the dissemination or wrecking a flexibly way to the brain. The mortality rate will rise for stroke in the coming years, according to the World Health Organization. Lots of research has been performed to diagnose stroke disease. To predict stroke and its type, we use an artificial intelligence approach that uses deep learning. An ischemic stroke, hemorrhagic stroke and a latent ischemic stroke are examples of this. Data collection in our research is from the Scientific Institute. The pre-processing system expels copy archives, missing data and contested knowledge. Guideline component examination estimation calculation is utilized to limit forecasts and deep learning by deciding if the individual is having a stroke.It changes the definition to anticipate the condition of a stroke by methods for profound learning. When the patient subtleties are entered it contrasts and the prepared model and predicts various sorts of stroke. This exploration centers essentially around a superior method of anticipating stroke and diverse stroke structures.
\end{abstract} Index terms- Artificial intelligence, artificial neural network
and K nearest neighbor algorithm.

\section{INTRODUCTION}

Stroke is a third basic reason for death and insufficiency for long haul. Stroke is a neurological issue emerging at a specific second. A coagulation happens in isolated veins or blood trenches. As the world health organization suggested, stroke will continue to increase the risk of death in the coming years. So, under the circumstances, severe treatment of the condition has to be as fast as intended. All things considered, one million individuals are confronting a stroke. Powerlessness includes strife disfigurement, vision misfortune, balance misfortune and voice misfortune. Stroke is a genuine perilous condition, as a respiratory failure that harms the heart. It doesn't deplete the blood, so furnishes the synapses with oxygen. Anybody beats the risk of a specific stroke. It can cause loss of parity, unexpected chest torment, voice barrenness, cognitive decline and thought limits, diminish state or passing. Stroke influences individuals of each age. It tends to be dealt with supportive guideline, and it is essential to change the hazard factors. The investigation expresses that the most recorded therapeutic mix-up exists as far as remedy expiry, messed up drugs, off base ends and care given to an inappropriate patient.

For the most part, three types of strokes can be found:

\author{
- Ischemic stroke \\ - Haemorrhagic stroke \\ - Transient ischemic stroke
}

\section{LITERATURE SURVEY}

Medicinal services offices additionally give different points of interest, for example, counteraction of extortion in human services protection, availability of open patient clinical administrations, acknowledgment of current clinical philosophies, and progression of inventive social insurance advances and viable emergency clinic asset the executives, improved client commitment, better patient consideration and emergency clinic disease control. Stroke type recognition is also a key field of medical research. Predicting stroke disease isn't automatic.

[1] In this work they compare various methods with our Cardiovascular Health Study (CHS) dataset approach for stroke prediction. In this case, the decision tree algorithm is used for the selection of features, the main component analysis algorithm is used to reduce the dimension, and the neural network classification algorithm adopted back propagation is used to create a classification model.

[2] In this paper, they contrast the Cox corresponding risks model and the Cardiovascular Health Study (CHS) informational index of an AI strategy for stroke expectation. In this report, we contrast the Cox relative dangers model and the Cardiovascular Health Report (CHS) informational collection of an AI way to deal with stroke expectation.

[3] This paper explores the various predictive models developed to date for stroke risk assessment. To predict the risk of stroke, various traditional statistical methods and computational intelligent models, such as the Framingham model, were developed. These models of risk prediction can help in clinical decision-making and help patients have an improved and reliable prediction of risk.

[4] The author utilized the fozzy congnitive guides and non-straight hebbian learning calculation in this paper to conjecture the likelihood of stroke.. Because in the performance tests the consumer used fuzzy congnitive maps with less precision. And the prediction of the likelihood of a Stroke will lead to less accurate results.

[5] In this paper they presented the concepts of data mining 
to retrieve the structures and algorithms. They used average property values for continuous features, and mean values. WEKA 3.6 has been used for analysis of the results. The J48 and IBK algorithms for implementing the C4.5 algorithm version 8 and the corresponding K-nearest algorithm.

[6] Machine learning for profound learning (man-made brainpower) procedures utilizing profound neural systems ( DNNs) has been executed in this examination to exceed expectations in working with complex sources of info. Primary territories for inevitable utilization of profound figuring out how to stroke the board are picture division, programmed featurization (radiomics), and multimodal visualization. Applying convolutionary neural systems, the group of DNN structures intended to work with pictures, stroke picture information is a decent match between a develop, profound learning procedure and an information structure that is obviously fit to profit by the qualities of profound learning.

[7] In this paper, the author proposed an in order symmetry counter-lateral algorithm to detect the stroke. The fundamental element of this calculation is to recognize the different reasons for stroke, for example, intense, interminable and hemorrhages. Here the calculation is utilized in a CT number to decide the cuts that are influenced by the stroke.

[8] A system for foreseeing the hazard pace of stroke dependent on fuzzy intellectual guides and nonlinear Hebbian learning calculation has been proposed in this paper. The precision of the proposed NHL-FCM model is assessed utilizing 15-overlap cross-approval, for 90 genuine cases, and contrasted with help vector machine and neighbors k-nearest.

[9] In this paper, an automated method for detecting and classifying an abnormality at the slice level of non- contrast CT images into acute infract and haemorrhage. Here it consists of three steps image enhancement, mid- line symmetry identification and anomalous slice classification.

[10] In this paper, physicians use CT to identify various forms of intracranial hematomas according to their positions and shapes, including epidural, subdural and intracerebral hematomas. Here they suggest a novel methodology that can consequently analyze intracranial hematomas by incorporating procedures for machine vision and the investigation of data. The skull is set on the CT cut, and the profundity of each intracranial pixel is named. The hyper thick territory of intracranial hematoma is portioned with multi-goal thresholding and district becoming after the pixel forces are standardized by their profundities.

\section{METHODOLOGY}

It's elusive a solid or even a well-performing AI calculation for a particular dataset when you fit the calculation to prepare the bundle. We've experienced an experimentation procedure to settle on a short rundown of calculations that produce better outcomes. We had taken in a couple of calculations. In our work we will use the $\mathrm{K}$ Nearest Neighbor algorithm to determine whether or not the person is suffering from a stroke and if a person is suffering it determines the type of stroke the person is suffering from.

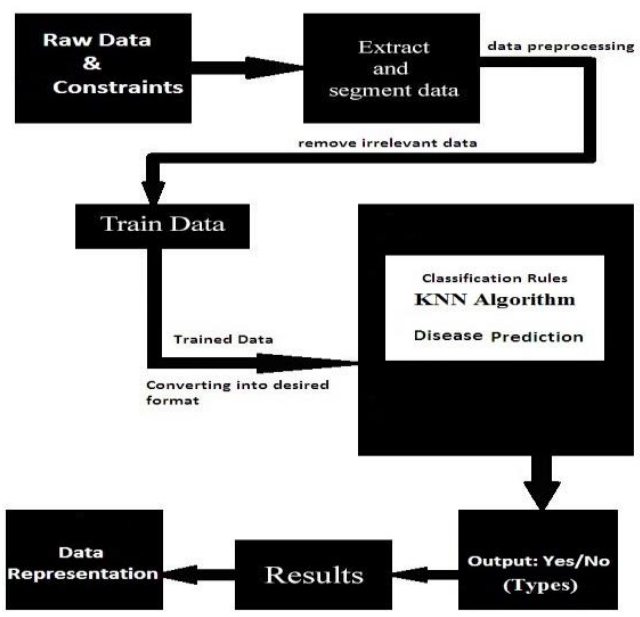

Fig(1): System Architecture

The block diagram of the structure being proposed is shown in the fig(1) above. Firstly, the data set undergoes the pre-processing stage where the irrelevant data is extracted and if the dataset contains any missing data or duplicate records then all those irrelevant data are extracted in this stage. And then the entire dataset will be translated to the desired format and the data set will be supplied to the algorithm as input. In this method we use a $\mathrm{KNN}$ algorithm to predict whether or not a person suffers from a stroke. The performance of the device will be " 0 " if a person is regular, "1" if a person has Ischemic stroke, "2" if a person has Hemorrhagic stroke and " 3 " if a person has Transient Ischemic stroke. To find the algorithm's accuracy the entire data set is split into two parts as a training data set with known results and test data set without results, but the known test data set results are taken as the actual data set. The training data set is used to train the program, and then we will find out the results of the test data which will then be compared with the real data set to determine the algorithm's accuracy.

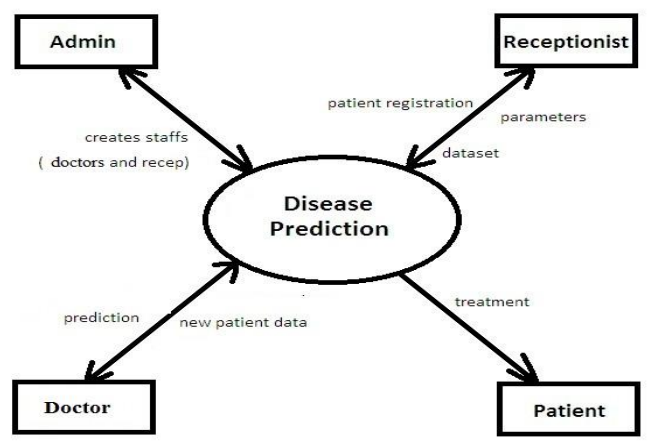

Context Flow Diagram - Level 0

Fig(2): Flow Chart 
A flowchart of the proposed system is shown in the diagram above. Four separate users will access the proposed program, and they are Admin, Receptionist, Doctor, and Patient. Admin is the one that manages the application as a whole and admin is the owner. Admin also establishes the staff such as doctors and receptionists and manages the staff and sets the unique Id and password for each staff member. Receptionist is one who retains information about patient registration, billing and care. Doctor is one who determines the inputs required for predicting disease. Doctor is a recipient for the service. The System's main feature is "Stroke Disease Prediction" based on medical evidence. The patient receives the care from the application, and can access the specifics of the treatment.

\section{RESULTS ANALYSIS}

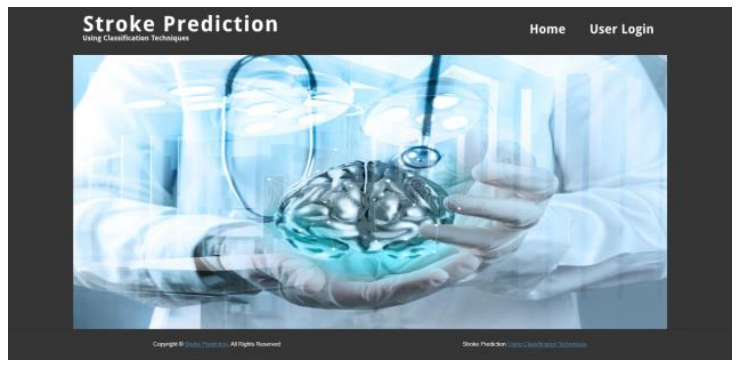

Fig(3): Home page

The fig(3) show the home page of our Stroke Prediction user page. In the right side of the page we provide a login credentials to all the four separate users they are Admin, Receptionist, Doctor, and Patient.

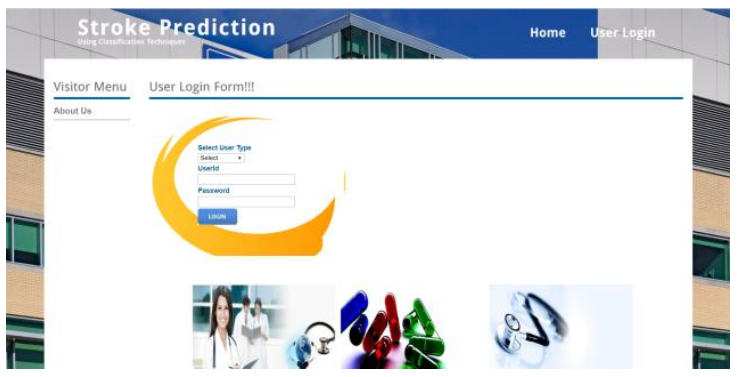

Fig(4): User Login page

The fig(4) describes user login credentials and Each individual user has its own user name and password. Here login process involves user authentication.

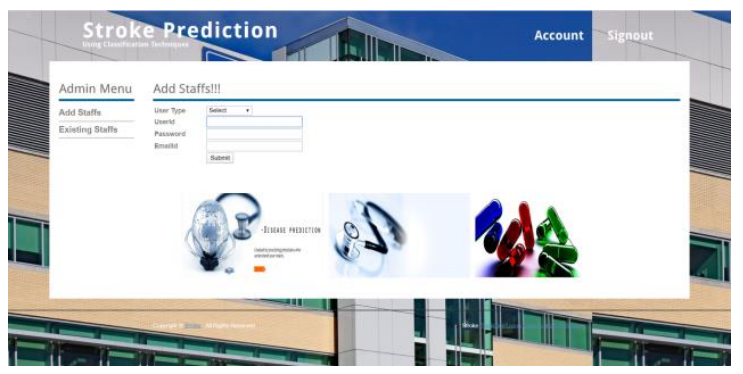

Fig(5): Admin Page to create staffs

The fig(5) shows that all user credentials will be created and removed by Admin. Only Admin has that credentials to change ones username and password.

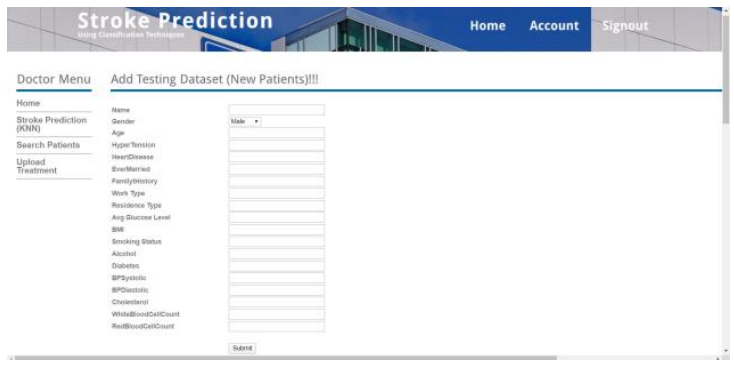

Fig(6): Doctor Page to add New Patients

The fig(6) describes that the Doctor must fill all the 18 parameters which will results on stroke prediction.

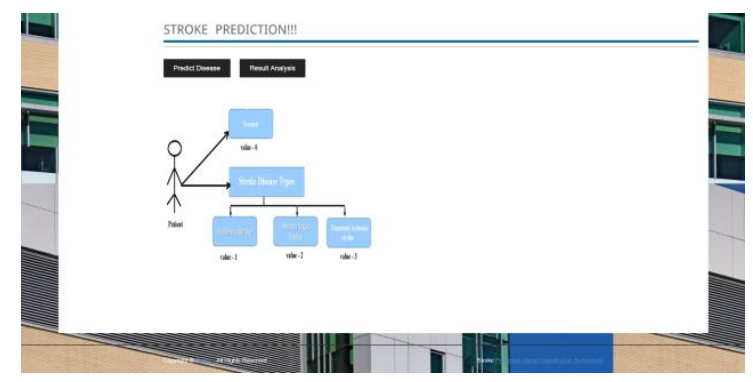

Fig(7):Result page

The fig(7) shows the result values associated with the type of the stroke.

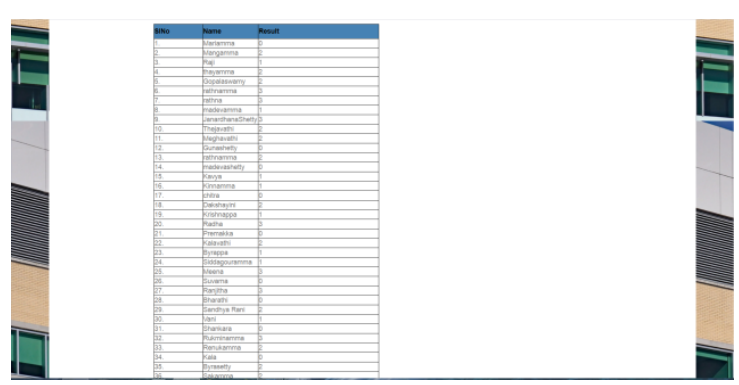

Fig(8):Prediction page

The fig(8) shows the results of the testing dataset

0. Normal

1. Ischemic stroke

2. Haemorrhagic stroke

3. Transient ischemic stroke

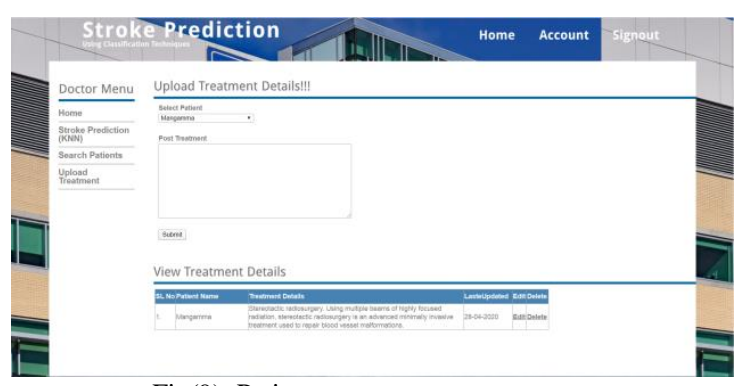

Fig(9): Patient user page

The fig(9) shows the treatment details uploaded by the doctor. 


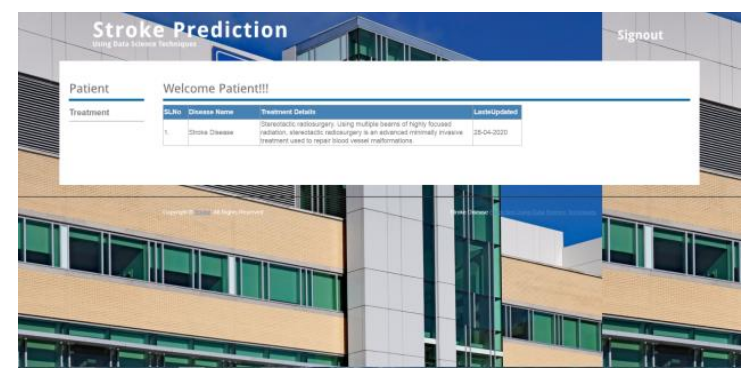

Fig(10): Treatment Details

The fig(10) shows the patient page which shows the treatment details.

\section{V.CONCLUSION}

Expectation type of the stroke has become a worldwide medical problem, and is a zone of concern. Present programming is a manual, tedious strategy that needs more, and costly, specialist experience. Most examinations were directed on the investigation of automated stroke, and its subtypes were on picture readiness techniques and CT checking and MRI. A fake neural system gives a general strategy to issue finding. To foresee stroke contamination it gets neural system for request count. The interpretation of stroke disease based on an artificial neural network, with greater accuracy, improves theoretical precision. The proposed framework is a stoke prediction algorithm which uses machine learning or AI techniques.

\section{REFERENCES}

[1] M.sheetal Singh, Prakash Choudhary; Stroke prediction using artificial intelligence IEEE paper published in 2017.

[2] Yu Cao, Hai Liang Chiu, Aditya Khosla and Cliff Chiung Yu Lin; A Machine Learning Approach to Stroke Risk Prediction.SFariborz Khorvash, Rasul Norouzi, "Prediction and Control of Stroke by DataMining".

[3] Jeena R S, Dr Sukesh Kumar A; Stroke Prediction Models : A Systematic Review.

[4] Pattanapong Chantamit-o-pas and Madhu Goyal, "Prediction of Stroke Disease using Deep Learning Model".

[5] Leila Amini, Reza Azarpazhouh, Mohammad Taghi Farzadfar, Sayed Ali Mousavi, Farahnaz Jazaieri, Fariborz Khorvash, Rasul Norouzi and Nafiseh Toghianfar; Prediction and Control of Stroke by Data Mining.

[6] Feng R, Badgeley M, Mocco J,Oermann EK "Deep learning guided stroke management: a review of clinical applications" 2018.

[7] D. Cosic and S. Loncaric, "Computer system for quantitative analysis of ich from ct head images" in $19^{\text {th }}$ Annual International Conference of the IEEE, 1997.

[8] Mahsa Khodadai, Heidarali Shayanfar, Keivan Maghooli ORCID Icon \& Amir Hooshang Mazinan "Prediction of stroke probability occurrence based on fuzzy cognitive maps" 2017.

[9] JoonNyunh Heo, Jihoon G. Yoon, Hyungjong Park, young Dae Kim, Hui suk nam, ji hoe "Machine Learning-Based model for prediction of outcomes in acute stroke".

[10] C.-C.Liao, F.Xiao, J-M. Wong and I-J. Chi-ang. A knowledge discovery approach to di-agnosing intracranial hematomas on brain ct: recognition, measurements and classification. In Medical Biometrics, pages 73-82. Springer,2007. 